\title{
Protocol and statistical analysis plan for the phase 3 randomised controlled Treatment of Invasively Ventilated Adults with Early Activity and Mobilisation (TEAM III) trial
}

Jeffrey J Presneill, Rinaldo Bellomo, Kathy Brickell, Heidi Buhr, Belinda J Gabbe, Doug W Gould, Meg Harrold, Alisa M Higgins, Sally Hurford, Theodore Iwashyna, Ary Serpa Neto, Alistair Nichol, Stefan J Schaller, Janani Sivasuthan, Claire Tipping, Steven Webb, Paul Young and Carol L Hodgson, for the TEAM Study Investigators and the Australian and New Zealand Intensive Care Society Clinical Trials Group

The quality of survival for patients after intensive care unit (ICU) admission is a major health challenge that has been described as the defining challenge for critical care in the 21st century. ${ }^{1} \mathrm{ICU}$ survivors may have delayed and compromised functional recovery, which can persist for months or years. 2,3 Preliminary evidence suggests that critically ill patients admitted to an ICU who are expected to require prolonged mechanical ventilation may benefit from early activity and mobilisation. ${ }^{4-6}$ The Treatment of Invasively Ventilated Adults with Early Activity and Mobilisation (TEAM III) trial (ClinicalTrials.gov identifier NCT03133377) is a phase 3 randomised controlled trial of patients who are expected to receive mechanical ventilation for more than 48 hours. In these patients, we will evaluate whether, compared with standard care, early mobilisation and rehabilitation commenced in the ICU increases the number of days alive and out of hospital at 180 days $\left(\mathrm{DAOH}_{180}\right)$ after randomisation. In this article, we describe the protocol and statistical analysis plan, which was finalised before completion of trial patient recruitment.

\section{Methods}

Study design and setting

TEAM III is an international, multicentre, prospective, parallel-group, randomised controlled phase 3 superiority trial evaluating the safety and efficacy of an early activity and mobilisation protocol compared with standard care. ${ }^{7}$ The first patient was randomly assigned in February 2018 and patient recruitment is estimated to be completed by October 2021. Final collection of all 180-day outcome data is anticipated by May 2022. The total recruitment target of 750 patients will be enrolled from sites in Australia, New Zealand, Germany, Ireland, the United Kingdom and Brazil.

\section{Study population}

The inclusion and exclusion criteria are shown in Table 1. Eligible patients will be randomly assigned to early activity

\begin{abstract}
Objective: To describe the protocol and statistical analysis plan for the Treatment of Invasively Ventilated Adults with Early Activity and Mobilisation (TEAM III) trial.

Design: An international, multicentre, parallel-group, randomised controlled phase 3 trial.

Setting: Intensive care units (ICUs) in Australia, New Zealand, Germany, Ireland, the United Kingdom and Brazil.

Patients: 750 adult patients expected to receive mechanical ventilation for more than 48 hours.

Interventions: Early activity and mobilisation delivered to critically ill patients in an ICU for up to 28 days compared with standard care.

Main outcome measures: The primary outcome is the number of days alive and out of hospital at 180 days after randomisation. Secondary outcomes include ICU-free days, ventilator-free days, delirium-free days, all-cause mortality at 28 and 180 days after randomisation, and functional outcome at 180 days after randomisation.

Results: Recruitment at 46 research sites passed 576 patients in March 2021. Final collection of all 180-day outcome data for the target of 750 patients is anticipated by May 2022.

Conclusions: Consistent with international guidelines, a detailed protocol and prospective analysis plan has been developed for the TEAM III trial. This plan specifies the statistical models for evaluating primary and secondary outcomes, defines covariates for adjusted analyses, and defines methods for exploratory analyses. Application of this protocol and statistical analysis plan to the forthcoming TEAM III trial will facilitate unbiased analyses of the clinical data collected.
\end{abstract}

Trial registration: ClinicalTrials. govidentifier NCT03133377. 


\section{ORIGINAL ARTICLE}

\section{Table 1. Inclusion and exclusion criteria}

\section{Inclusion criteria}

- Aged 18 years or older

- Intubated and expected to remain on invasive mechanical ventilation for more than 48 hours

- Sufficient cardiovascular stability to make mobilisation potentially possible, as indicated by:

- the absence of current bradyarrhythmia requiring pharmacological support

- a current ventricular rate $\leq 150$ beats/min

- most recent lactate level $\leq 4.0 \mathrm{mmol} / \mathrm{L}$

- current combined noradrenaline/adrenaline infusion rate of $\leq 0.2 \mu \mathrm{g} / \mathrm{kg} / \mathrm{min}$ or, if noradrenaline/adrenaline infusion rate has increased by more than $25 \%$ in the preceding 6 hours, dose must be $<0.1 \mu \mathrm{g} / \mathrm{kg} / \mathrm{min}$

- most recent cardiac index $\geq 2.0 \mathrm{~L} / \mathrm{min} / \mathrm{m}^{2}$ (where measured)

- no current requirement for venoarterial ECMO

- Sufficient respiratory stability to make mobilisation potentially possible, as indicated by:

- current $\mathrm{FiO}_{2} \leq 0.6$

- current positive end expiratory pressure $\leq 16 \mathrm{cmH}_{2} \mathrm{O}$

- an absence of current requirement for nitric oxide, prone ventilation, neuromuscular blockers, prostacyclin, venovenous ECMO or high-frequency oscillation ventilation

- current respiratory rate $\leq 45$ breaths/min

\section{Exclusion criteria}

- Dependent for activities of daily living in the month before the current ICU admission (gait aids are acceptable)

- Documented cognitive impairment

- Proven or suspected acute primary brain injury (eg, traumatic brain injury, stroke, hypoxic brain injury)

- Proven or suspected spinal cord injury or other neuromuscular disease that will result in permanent or prolonged weakness (not including ICU-acquired weakness)

- Has rest-in-bed orders and/or bilateral non-weight bearing orders for the lower limbs

- Life expectancy < 180 days owing to a chronic or underlying medical condition

- Death is deemed inevitable as a result of the current illness, and the patient, treating clinician or substitute decision maker are not committed to full active treatment

- Unable to communicate in the official local language

- This is not the first ICU admission in the index hospital admission

- Fulfilled all inclusion criteria and none of the exclusion criteria $\geq 72$ hours ago

$\mathrm{FiO}_{2}=$ fraction of inspired oxygen. $\mathrm{ICU}=$ intensive care unit. $E C M O=$ extra corporeal membrane oxygenation. and mobilisation, to the highest suitable level as assessed daily by a physiotherapist face to face during the ICU admission up to 28 days, or physiotherapy care that is standard for that hospital.

\section{Randomisation}

A web-based interface (https://www.teamtrial. org.au) will be used to confirm that patients fulfil all the inclusion criteria and no exclusion criteria. Randomisation between trial mobilisation and control interventions will be performed on this website using a computer-generated randomised treatment allocation schedule stratified by participating hospital site with a permuted scheme with blocks of varying sizes.

\section{Treatment masking (blinding)}

Patient mobility while receiving mechanical ventilation is obvious, so it is not possible to blind patients or clinical staff to treatment allocation. Bias will be minimised by concealed treatment allocation before randomisation, protocolised treatment of the intervention group, ${ }^{7}$ and assessment of the primary outcome by centralised, blinded and trained research staff in each region. In the United Kingdom, minimisation of bias will be augmented by data linkage. The primary outcome measure - number of $\mathrm{DAOH}_{180}$ after randomisation, with any days spent in a rehabilitation facility or nursing home counted as days in hospital - is subject to minimal ascertainment bias.

\section{Intervention}

We followed the UK's Medical Research Council guidelines for developing and evaluating a complex intervention and the template for intervention description and replication (TIDieR) checklist and guide (Online Appendix, D). ${ }^{8,9}$ Each patient allocated to the intervention group will receive physiotherapistdirected, functional rehabilitation comprising active exercises and mobilisation conducted as early as possible and at the highest level of ICU mobility scale activity possible for them (Figure 1; Online Appendix, E). ${ }^{10,11}$ The first full day will be considered Day 1 and the intervention will occur daily for the duration of the ICU stay up to Day 28. Each patient allocated to the control group will receive standard care from physiotherapy staff not involved in delivering the intervention, which in most cases will involve no active exercise out of bed. ${ }^{12}$ For both groups, concomitant care will be guided by the treating 


\section{ORIGINAL ARTICLE}

Figure 1. Intervention protocol

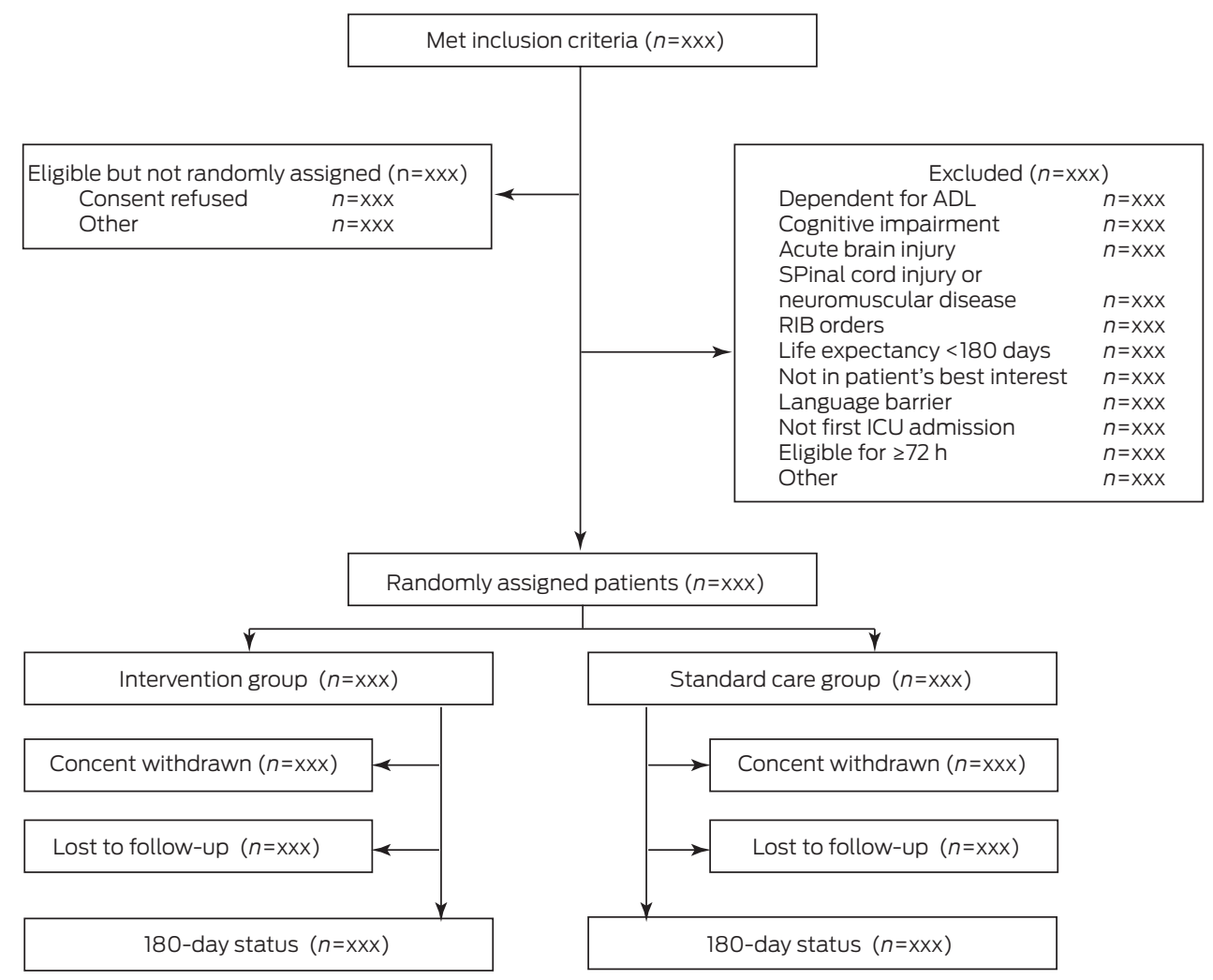

IMS = intensive care unit mobility scale.

clinician and all post-ICU patient management will be at the discretion of the patient's ward-based treating physicians. The intervention and study tools were designed to facilitate updates to clinical practice if the TEAM III trial results support such implementation. ${ }^{13}$

\section{Primary outcome}

The trial primary outcome variable will be $\mathrm{DAOH}_{180}{ }^{14-16}$ For each patient, days during the index hospitalisation, hospital readmission, inpatient rehabilitation or in a nursing home between randomisation and Day 180 will be subtracted from 180 to calculate their $\mathrm{DAOH}_{180}$ result (Table 2). Patients who die before Day 180 will be defined as having the worst possible outcome of zero $\mathrm{DAOH}_{180}$.

\section{Secondary outcomes}

Secondary outcome measures will be evaluated at 28 days and 180 days after randomisation (Table 2). Outcomes at Day 28 include: all-cause mortality; ICU-free days; ventilatorfree days; and delirium-free days measured using the
Confusion Assessment Method for the Intensive Care Unit (CAM-ICU) and Richmond Agitation-Sedation Scale (RASS). Patients who die before Day 28 will be assigned zero ICUfree, ventilator-free and delirium-free days. Outcomes at Day 180 include: all-cause mortality; time to death; DAOH in patients who die before Day 180; $\mathrm{DAOH}$ in patients alive at Day 180; days in hospital, a rehabilitation facility or a nursing home to Day 180 according to 180 -day survival status; quality of life and health status measured using the five-level EuroQol five dimensions questionnaire (EQ-5D$5 \mathrm{~L})$; independent activities of daily living scores measured using the Barthel activities of daily living ( $A D L$ ) index and the Lawton instrumental activities of daily living (IADL) scale; generic function and disability measured using the 12-level World Health Organization Disability Assessment Schedule 2.0 (WHODAS 2.0 12L); cognitive function measured using the Montreal Cognitive Assessment (MOCA)-Blind; and psychological function measured using the Hospital Anxiety and Depression Scale (HADS) and Impact of Event Scale Revised (IES-R). 


\section{ORIGINAL ARTICLE}

Table 2. Primary and secondary outcomes

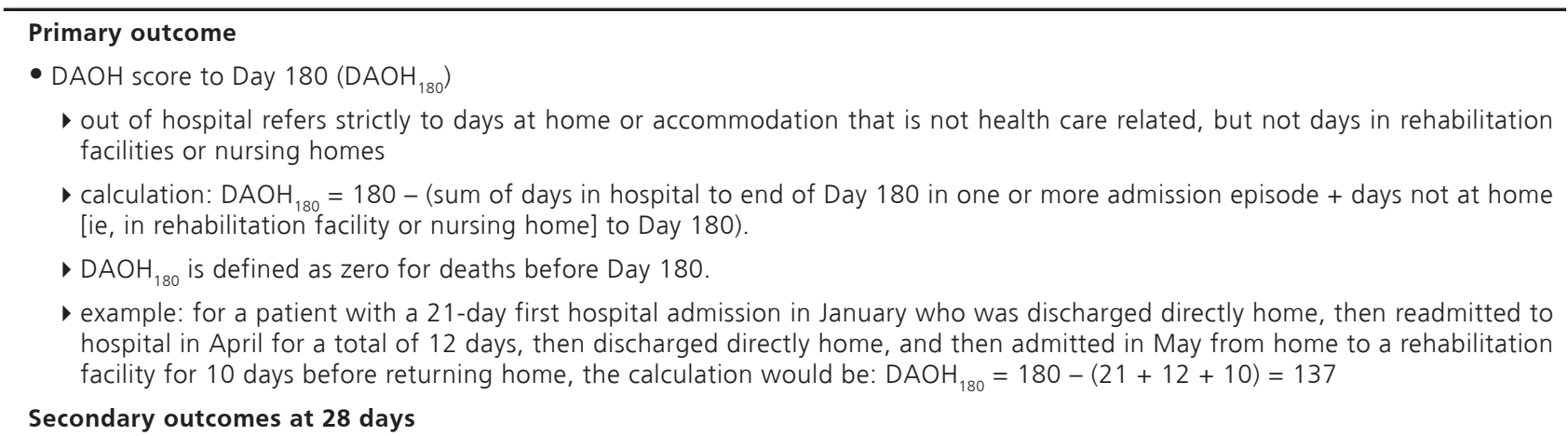

- ICU-free days from randomisation, defined as days alive and discharged from ICU*

- Ventilator-free days from randomisation, where extubation for $>48$ hours is successful ${ }^{+}$

- Delirium-free days (CAM-ICU and RASS score, from randomisation, censored at ICU discharge) ${ }^{\ddagger}$

- Time from randomisation until death

- Process-of-care measures

- tracheostomy in ICU

- neuromuscular blockers by continuous infusion

- reintubation during index ICU admission

- vasopressor-free days during index ICU admission from randomisation

- corticosteroids during index ICU admission

- new post-randomisation renal replacement therapy in ICU

- daily RASS sedation score

- ICU mobilisation outcomes (censored at ICU discharge if before Day 28)7,11

- highest daily ICU mobility scale in ICU

- total duration of active mobilisation in ICU

\section{Secondary outcomes at 180 days}

- All-cause mortality

- DAOH in patients who die before Day 180

- DAOH in patients alive at Day 180

- Time from randomisation until death

- Time from randomisation to ICU and hospital discharge, overall, and with death as a competing outcome

- ICU-free days, defined as days alive and discharged from ICU*

- Ventilator-free days from randomisation, where extubation for $>48$ hours is successful $^{+}$

- Quality of life and physical function

- EQ5D-5L score 17,18

- generic function and disability score (WHODAS $2.012 \mathrm{~L})^{19,20}$

- independent activities of daily living scores (Barthel ADL index and Lawton IADL scale) 21,22

- Cognitive and psychological function

- MOCA-Blind score 3,23

- hADS score 24

- IES-R score 25

\section{Economic outcome}

- Cost-effectiveness at 6 months

$A D L=$ activities of daily living. $C A M-I C U=$ Confusion Assessment Method for the Intensive Care Unit. DAOH = days alive and out of hospital. EQ5D$5 \mathrm{~L}=$ five-level EuroQol five dimensions questionnaire. HADS = Hospital Anxiety and Depression Scale. ICU = intensive care unit. IES-R $=$ Impact of Event Scale - Revised. IADL = instrumental activities of daily living. MOCA-Blind = Montreal Cognitive Assessment - Blind. RASS = Richmond Agitation-Sedation Scale. WHODAS 2.0 12L = 12-level World Health Organization Disability Assessment Schedule 2.0. * Patients who die at any time are assigned zero ICU-free days. + Patients who die at any time are assigned zero ventilator-free days. ₹ Patients who die at any time are assigned zero delirium-free days. 


\section{ORIGINAL ARTICLE}

\section{Statistical analyses}

Effect estimates for all outcomes will be accompanied by a $95 \%$ confidence interval $(\mathrm{Cl})$, consistent with a twosided type 1 error of $5 \%$. There will be no adjustment for multiplicity of testing among effect estimates and corresponding $95 \%$ Cls for non-primary outcomes, which therefore should be interpreted cautiously as hypothesisgenerating results. ${ }^{26}$ Descriptive and summary statistics will be calculated by treatment group and overall. Continuous data will be summarised as means (standard deviations [SDs]) if about normally distributed, and otherwise as medians (interquartile ranges). Categorical data will be summarised as counts and proportions. Unadjusted differences between groups will be assessed initially using two-sample $t$ tests or Wilcoxon rank-sum tests, or Fisher exact tests for proportions, as appropriate. The number of screened patients who fulfil the inclusion criteria, the numbers included in the primary and secondary analyses and all reasons for exclusion from the primary and secondary analyses will be reported according to CONSORT guidelines (Figure 2). The full analysis set for the main primary and secondary analyses will retain participants in their original randomly assigned groups, and include all randomly assigned patients except those who withdraw consent for use of trial data. 27,28 Wherever possible and appropriate, statistical inference will account for potential dependency in patient clusters formed by each participating ICU or hospital site. Linear and generalised linear model diagnostics, outlier assessment and remedial measures will follow standard approaches. $^{29,30}$ Proportional hazards assumptions across treatment arms in time-to-event analyses will be evaluated using scaled Schoenfeld residuals and visual assessment of log-log plots. ${ }^{31}$ Whenever needed, the proportional odds assumption for cumulative logistic models will be assessed

\section{Figure 2. Consolidated Standards of Reporting Trials (CONSORT) diagram for the TEAM III trial}

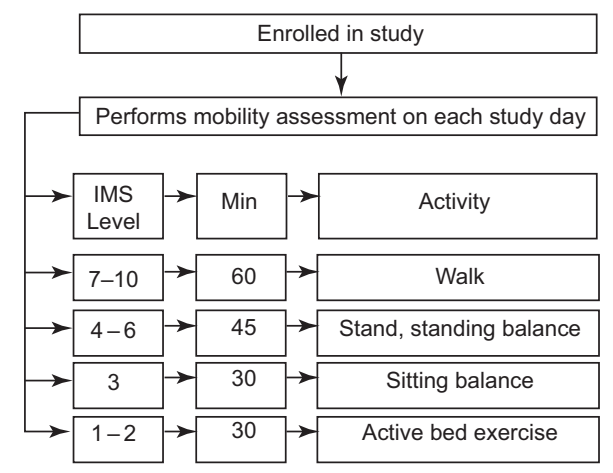

$A D L=$ activities of daily living. $I C U=$ intensive care $u$ nit. $R I B=$ rest in bed. by visual assessment of relevant plots for clinically important departures from proportionality.

\section{Primary outcome}

The primary outcome, $\mathrm{DAOH}_{180}$, is expected to have a bimodal and asymmetric frequency distribution. A linear quantile regression analysis will be used because this approach models selected conditional quantiles of a continuous response. ${ }^{32}$ Also, this is potentially more robust to outliers and misspecification of an error distribution compared with traditional least squares linear (mean) regression. $\mathrm{DAOH}_{180}$ will be modelled as a continuous random variable, with a null hypothesis of equality in the median (quantile $=0.5$ ) $\mathrm{DAOH}_{180}$ between the full analysis set of patients according to randomised treatment group. The magnitude of the differential primary outcome according to randomised treatment group will be derived from the treatment group effect estimate returned by a simple linear median quantile model with a term for binary treatment group. The number needed to treat for benefit or harm will also be reported if strong evidence of a difference between treatment groups is found. Model fitting will use published software for linear quantile models. At the time of writing, within the $R$ statistical environment, the quantile regression package quantreg offers a bootstrapped procedure for construction of cluster-robust standard errors with associated $95 \% \mathrm{Cls}$ to support cluster-robust inference (Online Appendix, 2 Calculation of primary outcome). ${ }^{33-35}$ Specification of either simplex (exterior point) or interior point median regression methods showed satisfactory relative power compared with several alternative analysis methods in simulated data scenarios (Online Appendix, 3 - Relative power of alternative methods for analysis of the primary outcome).

\section{Sensitivity analyses of the primary outcome}

Baseline covariate imbalance. In the event of baseline variable imbalance $(P<0.05)$ between randomisation arms despite the randomisation process, a sensitivity analysis of the primary outcome will be used to report the effect of the trial intervention on median $\mathrm{DAOH}_{180}$, with unbalanced baseline variable(s) included as fixed term(s) with the treatment term within that supplementary linear quantile regression (Online Appendix, 2 - Calculation of primary outcome). ${ }^{36}$

Missing data. If more than $5 \%$ of the primary outcome data are missing, complete case analyses will be accompanied by further sensitivity analyses using two SD best-worst case, two SD worst-best case, and multiple imputation under the assumption of data missing at random. ${ }^{37-40}$

Grid of quantiles. The quantile regression TEAM intervention effect estimate, adjusted according to the covariate(s) included in the primary outcome model, will 


\section{ORIGINAL ARTICLE}

be computed on a selected discrete set of quantiles within the $0.01 \leq \tau \leq 0.99$ range to summarise any non-constant TEAM intervention effect within the $\mathrm{DAOH}_{180}$ upper and lower tails. These results will be reported graphically and/ or in tables that also show, for comparison, the mean TEAM intervention effect estimate across all quantiles returned from a similarly constructed linear mixed-effect model. $35,40,41$

Results at each research site. The differential primary outcome according to randomised treatment group will be tabulated according to research site.

Ordinal categorical analysis. The differential effect of treatment will be estimated within a proportional odds cumulative logistic model treating $\mathrm{DAOH}_{180}$ as an ordinal categorical random variable. ${ }^{42}$ The wide range of $\mathrm{DAOH}_{180}$ categories may be collapsed to a convenient and clinically meaningful smaller number of ordered categories without important loss of statistical information. ${ }^{43-45}$

\section{Secondary outcomes}

Because no adjustment for multiple comparisons will be performed in the analyses of trial secondary outcomes, these outcomes and their reported unadjusted $95 \% \mathrm{Cls}$ will be considered hypothesis-generating. ${ }^{26}$

Time-to-event analyses. The time to events of interest, including mortality to 180 days, will be assessed using Kaplan-Meier plots and log-rank tests. These will be supplemented by Cox proportional hazard regression models including variables as for the primary outcome model, with the proportional hazards assumption assessed using Schoenfeld residuals, ${ }^{31}$ as described above. The time to discharge according to treatment group will be assessed using a competing risks model with death before discharge treated as a competing risk. ${ }^{46}$

Other binary outcomes. Other binary variables, including adverse events, will be analysed with logistic regression models to estimate odds ratios with $95 \% \mathrm{Cls}$.

Pre-specified subgroup analyses of the primary outcome. Possible heterogeneity of treatment effect in the following pre-specified subgroups will be evaluated by tests of interaction between each subgroup and the study treatment in the models described above: 47

- baseline WHODAS 2.0 category (no and mild disability versus moderate, severe and complete disability);

- age (dichotomised at the full analysis set median age);

- diagnosis (sepsis versus trauma versus other);

- severity of illness (dichotomised at the full analysis set median APACHE [Acute Physiology and Chronic Health Evaluation] III score); and

- frailty (a binary reduction of the seven-point Clinical Frailty Scale at 1-4 [well and vulnerable] versus 5-7 [frail]). ${ }^{48}$
The new appearance of severe acute respiratory syndrome coronavirus 2 (SARS-CoV-2) infection across the world during the coronavirus disease 2019 (COVID-19) pandemic introduces the possibility that these patients may be enrolled in TEAM III in substantial numbers. These patients may have experienced a different clinical trajectory from other ICU patients recruited so far, including prolonged durations of mechanical ventilation accompanied by days of pharmacological paralysis and treatment with glucocorticoid medication. ${ }^{42,49}$ Using the full analysis set, the primary outcome will also be evaluated according to SARS-CoV-2/COVID-19 status subgroup (known positive versus negative or unknown).

Outcomes at $\mathbf{2 8}$ days. Outcomes at 28 days are listed in Table 2. The scores of ICU-free, ventilator-free and deliriumfree days evaluated at 28 days after randomisation will be modelled as continuous random variables in the same way as for the primary analysis. These scores will be zero for patients who die before Day 28.

Health-related quality of life. Health-related quality of life (HRQOL), health problems in each domain of the EQ-5D$5 \mathrm{~L}$ and functional outcomes assessed at 180 days will be summarised as means (with SDs), medians (with interquartile range) or as proportions. Unadjusted differences in HRQOL between groups will be assessed initially using the twosample $t$ test or Wilcoxon rank-sum test, or the Fisher exact test for proportions, as appropriate. HRQOL measures will further be evaluated using multivariable linear regression (or quantile regression where normality assumptions are violated) with adjustment for clustering by site and potential baseline imbalance $(P<0.05)$. These outcomes include the EQ-5D-5L utility score, EuroQol visual analogue scale (EQVAS) score (range, 0-100), ADL score (range, 0-20), IADL score (range, 0-8), WHODAS $2.012 \mathrm{~L}$ score (percentage), MOCA-Blind score (range, 0-22), HADS score (range, 0-21 for either anxiety or depression), and the IES-R score (range, 0-88). EQ-5D-5L utility scores will be calculated using an Australian or UK value set if available at the time of analysis, or a crosswalk value set where an appropriate EQ-5D-5L value set is not available. The WHODAS 2.0 12L percentage scores will be used to categorise patients into five mutually exclusive disability categories at 180 days: no disability (0-4\%); mild disability (5-24\%); moderate disability (25-49\%); severe disability (50-95\%); and complete disability (96-100\%). ${ }^{50}$ In addition, patients will be classified as having no or mild disability if their WHODAS score was $<25 \%$, and classified as moderate, severe or complete disability if their WHODAS score was $\geq 25 \%$.

Cost-effectiveness analyses. Cost-effectiveness analyses will be conducted from a health care payer perspective using 


\section{ORIGINAL ARTICLE}

a within-trial time horizon. Incremental cost-effectiveness ratios will be calculated as a cost per additional qualityadjusted life-year and cost per additional day alive and out of hospital gained at Day 180 for early activity and mobilisation compared with standard care. The EQ-5D-5L, administered 6 months after randomisation, will enable utilities to be determined and subsequent calculation of quality-adjusted life-years (QALYs). Costs will be determined based on resource use during the intensive care, acute care and post-acute care periods up to Day 180 and will include costs of the intervention where appropriate. To address the issue of transferability in multinational trials, we will adjust for heterogeneity across geographic regions (which may arise, for example, owing to differences in treatment patterns) and obtain estimates for costs and effects in specific regions of the study.

All costs will be converted to a common currency using purchasing power parity statistics from the Organisation for Economic Co-operation and Development. Health care costs will be compared between regions using the same methods as used in previous economic evaluations of multinational clinical trials by our group. 51

Per-protocol analyses. It is well understood that trial protocols may not be followed fully for some trial participants. ${ }^{52}$ Following completion of the main primary and secondary outcome analyses, there will be an option to repeat a selection of those analyses using a per-protocol analysis set, whereby the subset of protocol-compliant patients assigned to early activity and mobilisation is compared with all patients assigned to the control group. ${ }^{7,27,53}$ Protocol-compliant patients will be determined retrospectively; this will exclude patients for whom there were major protocol violations (eg, violation of entry criteria leading to random assignment of ineligible patients), patients who were not assessed by a physiotherapist for possible early activity and mobilisation on every day from randomisation to ICU discharge (to a maximum of 28 days), and patients for whom the primary outcome variable is not available.

\section{Data management}

All trial data will be collected by trained staff at each participating site and entered into a secure database (using electronic case report forms) by site staff using a web interface. Data management will be coordinated by project managers at the Australian and New Zealand Intensive Care (ANZIC) Research Centre, including programming (online study database design) and data management support (data monitoring, database questions, technical issues, data queries, query resolution). Data collection methods are detailed in the Online Appendix, E. All numerical data fields in the trial database have upper and lower review limits based on biologically unlikely thresholds to help identify possible data entry errors. Also, all data entered by European Union (EU) countries will be pseudonymised to align with EU data regulations.

Study monitors from the ANZIC Research Centre, the Medical Research Institute of New Zealand and Intensive Care National Audit and Research Centre in the UK will monitor all research sites in person, with the assistance of a German-speaking trial monitor at sites in Germany, a Portuguese-speaking monitor at sites in Brazil and a trial monitor from the Irish Critical Care Clinical Trials Network at sites in Ireland. During monitoring visits, multiple aspects of data validity are checked, including consent documents, inclusion and exclusion criteria, adverse events, and all admission and discharge dates required to calculate primary outcome data and important daily data. An independent data and safety monitoring committee (DSMC) continues to oversee the quality of the trial and has access to trial outcome and accumulated safety data, including the differential proportions of total mortality. Further details are available in the Online Appendix, C.

\section{Sample size, power and interim analysis schedule}

Conservative inflation factors were applied to a standard parametric calculation for sample size in this quantile regression. Estimated mean and SD DAOH ${ }_{180}$ were derived from a published pilot TEAM randomised controlled trial and systematic review, assuming overall mean \pm SD days alive and out of hospital of $143 \pm 21$ (control group) versus $156 \pm 27$ (intervention group). 7,16 Using a type I error proportion of 0.05 and a 7-day difference between the groups in $\mathrm{DAOH}_{180}$ (being about half the point estimate found in the pilot randomised controlled trial), recruiting 313 patients in each group would achieve $90 \%$ power to detect a difference. The recruitment goal was set at 750 patients. This final trial size incorporated an inflation of $15 \%$ to account for a non-parametric distribution of the primary end point and up to a $5 \%$ loss to follow-up; in comparison, previous studies by the ANZICS Clinical Trials Group have yielded losses to follow-up of $\leq 2 \% .{ }^{54,55}$

\section{Interim analysis}

There has been one interim analysis assessing efficacy and safety by the DSMC located in Ireland, the UK and the US, with no early stopping option planned for futility. A time point of 1 month after the first 400 patients reached their 28-day follow-up (3 June 2020) was set so that the interim analysis was conducted in a timely manner. At that point, the DSMC had access to:

- differential all-cause mortality at hospital discharge, censored at Day 28; 


\section{ORIGINAL ARTICLE}

- the differential number of days alive and not in hospital to Day 28 , with a score of zero allocated to any survivors who remained in hospital at Day 28; and

- the differential number of days alive and not in hospital to Day 180 for those with available data.

The DSMC's interim evaluation of Day 28 censored accumulated differential all-cause mortality at hospital discharge was supported by symmetrical \pm 3 SD HaybittlePeto boundaries, with this interim analysis "spending" a total of 0.0031 of the available type 1 error at that point. ${ }^{56}$ To preserve an overall type 1 error no greater than 0.05 , the final analysis of the primary trial outcome at full recruitment should be conducted at a two-sided $P$ value of 0.0491 $(Z= \pm 1.967)$, which in practice will be assumed unchanged from 0.05. ${ }^{57}$ On 2 September 2020, the DSMC confirmed that the TEAM III trial was approved to continue recruitment to the final target sample size.

\section{Analysis software}

Data capture and processing will initially be done by Research Path. Data will then be exported in relevant formats for statistical analyses using current versions of $R$ software, SAS software or Stata software. ${ }^{33,58,59}$

\section{Safety and adverse event analyses}

Safety and tolerability implications will be summarised using descriptive statistical methods, supplemented by calculation of $95 \% \mathrm{Cls}$ where appropriate. Patients with protocol deviations, adverse events and missing values will be identified, and a descriptive analysis that includes their relationship to treatment will be undertaken.

\section{Ethics and informed consent}

Ethics approval for the study was obtained from the responsible local and national human research ethics committees before recruitment at each study site commenced. By virtue of the inclusion criteria, none of the patients who are eligible for this study will be competent to provide prospective informed consent. The approach to consent in this study will be based on that developed from the guidelines in Chapter 4.4 of the 2013 edition of the National Health and Medical Research Council's National statement on ethical conduct in human research. Informed consent to participate will comply with requirements of relevant local and national human research ethics committees.

\section{Compliance with Good Clinical Practice requirements}

The trial is being conducted, and accumulating data are being monitored, according to the standard requirements of Good Clinical Practice. ${ }^{60}$

\section{Discussion}

Invasive mechanical ventilation is a life-saving intervention, but patients receiving this intervention are typically confined to bed with no active exercise. This immobilisation may contribute substantially to the development of muscle weakness and wasting in many such patients, with these undesirable events associated with increased hospital length of stay, increased mortality after hospital discharge, and poor long-term functional recovery. At present, there are no specific proven therapies available for this common clinical scenario.

The potential benefits of a bundle of activities comprising early activity and mobilisation during prolonged invasive mechanical ventilation are supported by scientific rationale. This intervention has the potential to shorten ventilator dependence, decrease mortality, and reduce physical and cognitive functional decline associated with illnesses needing prolonged mechanical ventilation in an ICU.

The TEAM III trial is designed to detect an important beneficial effect of early activity and mobilisation, if one exists, while minimising potential risks. Application of the statistical analysis plan within this TEAM III trial protocol will facilitate evaluation of these important clinical data and support confidence in the subsequent generalisation of its findings. The aim of the TEAM III trial is to provide definitive guidance for clinicians regarding the true efficacy and safety of a bundle of activities comprising early activity and mobilisation in the management of critical illness in adults.

\section{Trial status}

The TEAM III trial began in November 2017, with patient recruitment estimated to be completed by about October 2021 with a total of 750 participants, and final collection of all 180-day outcome data anticipated by May 2022. The trial recruitment at a total of 46 research sites passed 576 patients in March 2021.

Acknowledgements: This investigator-initiated research is funded in Australia by the National Health and Medical Research Council (GTN 1120319) and in New Zealand by the Health Research Council of New Zealand (GTN 19/021). Trial design, data collection and analyses are being undertaken independent of the funding bodies. Trial coordination is being led by the Australian and New Zealand Intensive Care Research Centre, part of Monash University in Melbourne, in collaboration with the Medical Research Institute of New Zealand and the UK's Intensive Care National Audit and Research Centre. The trial is endorsed by the Australian and New Zealand Intensive Care Society Clinical Trials Group. The members of the TEAM management committee 


\section{ORIGINAL ARTICLE}

and the TEAM trial sites and investigators are listed in the Online Appendix, $A$ and $B$ respectively).

\section{Competing interests}

No relevant disclosures.

\section{Author details}

Jeffrey J Presneill ${ }^{1,2}$

Rinaldo Bellomo ${ }^{1,3}$

Kathy Brickell ${ }^{4}$

Heidi Buhr ${ }^{5}$

Belinda J Gabbe 6

Doug W Gould 7

Meg Harrold 8,9

Alisa M Higgins

Sally Hurford ${ }^{10}$

Theodore Iwashyna ${ }^{11,12}$

Ary Serpa Neto ${ }^{1,13}$

Alistair Nichol ${ }^{1,4}$

Stefan J Schaller ${ }^{14}$

Janani Sivasuthan ${ }^{1}$

Claire Tipping ${ }^{15}$

Steven Webb ${ }^{1,16}$

Paul Young 11,12,17

Carol L Hodgson 1,15

For the TEAM Study Investigators and the Australian and New

Zealand Intensive Care Society Clinical Trials Group

1 Australian and New Zealand Intensive Care Research Centre, School of Public Health and Preventive Medicine, Monash University, Melbourne, VIC, Australia.

2 Department of Critical Care, University of Melbourne and Royal Melbourne Hospital, Melbourne, VIC, Australia.

3 Critical Care Department, Austin Hospital, Melbourne, VIC, Australia.

4 University College Dublin Clinical Research Centre, St Vincent's University Hospital, Dublin, Ireland.

5 Royal Prince Alfred Hospital, Sydney, NSW, Australia.

6 School of Public Health and Preventive Medicine, Monash University, Melbourne, VIC, Australia.

7 Intensive Care National Audit and Research Centre, London, UK.

8 Curtin University, Perth, WA, Australia.

9 Royal Perth Hospital, Perth, WA, Australia.

10 Medical Research Institute of New Zealand, Wellington, New Zealand.

11 Division of Pulmonary and Critical Care Medicine, Department of Internal Medicine, University of Michigan, Ann Arbor, Michigan, USA.

12 VA Center for Clinical Management Research, VA Ann Arbor Healthcare System, Ann Arbor, Michigan, USA.
13 Department of Critical Care Medicine, Hospital Israelita Albert Einstein, Sao Paulo, Brazil.

14 Department of Anesthesiology, Division of Operative Intensive Care Medicine, Charité - Universitätsmedizin Berlin, Humboldt Universität zu Berlin and Freie Universität Berlin, Berlin, Germany.

15 Department of Physiotherapy, Alfred Health, Melbourne, VIC, Australia.

16 St John of God Subiaco Hospital, Perth, WA, Australia.

17 Intensive Care Unit, Wellington Hospital, Wellington, New Zealand.

Correspondence: carol.hodgson@monash.edu

doi: https://doi.org/10.51893/2021.3.0A3

\section{References}

1 Iwashyna TJ. Survivorship will be the defining challenge of critical care in the 21st century. Ann Intern Med 2010; 153: 204-5.

2 Herridge MS, Tansey CM, Matte A, et al. Functional disability 5 years after acute respiratory distress syndrome. $N$ Engl J Med 2011; 364: 1293-304.

3 Iwashyna TJ, Ely EW, Smith DM, Langa KM. Long-term cognitive impairment and functional disability among survivors of severe sepsis. JAMA 2010; 304: 1787-94.

4 Adler J, Malone D. Early mobilization in the intensive care unit: a systematic review. Cardiopulm Phys Ther J 2012; 23: 5-13.

5 Zhang L, Hu W, Cai Z, et al. Early mobilization of critically ill patients in the intensive care unit: a systematic review and meta-analysis. PLoS One 2019; 14: e0223185.

6 Hodgson CL, Stiller K, Needham DM, et al. Expert consensus and recommendations on safety criteria for active mobilization of mechanically ventilated critically ill adults. Crit Care 2014; 18: 658.

7 Hodgson CL, Bailey $\mathrm{M}$, Bellomo $\mathrm{R}$, et al. A binational multicenter pilot feasibility randomized controlled trial of early goal-directed mobilization in the ICU. Crit Care Med 2016; 44: 1145-52.

8 Craig P, Dieppe P, Macintyre S, et al. Developing and evaluating complex interventions: the new Medical Research Council guidance. BMJ 2008; 337: a1655.

9 Hoffmann TC, Glasziou PP, Boutron I, et al. Better reporting of interventions: template for intervention description and replication (TIDieR) checklist and guide. BMJ 2014; 348: g1687.

10 Tipping CJ, Bailey MJ, Bellomo R, et al. The ICU Mobility Scale has construct and predictive validity and is responsive. A multicenter observational study. Ann Am Thorac Soc 2016; 13: 887-93.

11 Hodgson C, Needham D, Haines K, et al. Feasibility and interrater reliability of the ICU Mobility Scale. Heart Lung 2014; 43: 19-24.

12 Team Study Investigators; Hodgson C, Bellomo R, et al. Early mobilization and recovery in mechanically ventilated patients 


\section{ORIGINAL ARTICLE}

in the ICU: a bi-national, multi-centre, prospective cohort study. Crit Care 2015; 19: 81

13 Australian Clinical Trials Alliance. Guidance on implementability. Melbourne: ACTA, 2019.

14 Myles PS, Shulman MA, Heritier S, et al. Validation of days at home as an outcome measure after surgery: a prospective cohort study in Australia. BMJ Open 2017; 7: e015828.

15 Jerath A, Austin PC, Wijeysundera DN. Days alive and out of hospital: validation of a patient-centered outcome for perioperative medicine. Anesthesiology 2019; 131: 84-93.

16 Tipping CJ, Harrold M, Holland A, et al. The effects of active mobilisation and rehabilitation in ICU on mortality and function: a systematic review. Intensive Care Med 2017; 43: 171-83.

17 Cuthbertson BH, Elders A, Hall S, et al. Mortality and quality of life in the five years after severe sepsis. Crit Care 2013; 17: R70.

18 Dowdy DW, Eid MP, Dennison CR, et al. Quality of life after acute respiratory distress syndrome: a meta-analysis. Intensive Care Med 2006; 32: 1115-24.

19 Hodgson CL, Udy AA, Bailey $M$, et al. The impact of disability in survivors of critical illness. Intensive Care Med 2017; 43: 992-1001.

20 Ustun TB, Chatterji S, Kostanjsek N, et al. Developing the World Health Organization Disability Assessment Schedule 2.0. Bull World Health Organ 2010; 88: 815-23.

21 Hopkins RO, Suchyta MR, Kamdar BB, et al. Instrumental activities of daily living after critical illness: a systematic review. Ann Am Thorac Soc 2017; 14: 1332-43.

22 Lawton MP, Brody EM. Assessment of older people: selfmaintaining and instrumental activities of daily living. Gerontologist 1969; 9: 179-86.

23 Pandharipande PP, Girard TD, Jackson JC, et al. Long-term cognitive impairment after critical illness. N Engl J Med 2013; 369: 1306-16.

24 Rabiee A, Nikayin S, Hashem MD, et al. Depressive symptoms after critical illness: a systematic review and meta-analysis. Crit Care Med 2016; 44: 1744-53.

25 Weiss DS. The Impact of Event Scale-Revised. In: Wilson JP, Keane TM, editors. Assessing psychological trauma and PTSD: a practitioner's handbook. 2nd ed. New York: Guilford Press, 2004.

26 US Food and Drug Administration. Multiple endpoints in clinical trials: guidance for industry. Silver Spring: FDA, 2017. https:// www.fda.gov/media/102657/download (viewed July 2021).

27 International Council on Harmonisation. ICH harmonised guideline: addendum on estimands and sensitivity analysis in clinical trials to the guideline on statistical principles for clinical trials (ICH E9(R1)). https://database.ich.org/sites/default/files/ E9-R1_Step4_Guideline_2019_1203.pdf (viewed July 2021).

28 Schulz KF, Altman DG, Moher D; CONSORT Group. CONSORT 2010 statement: updated guidelines for reporting parallel group randomised trials. BMJ 2010; 340: c332.

29 Hosmer DW, Lemeshow S, Sturdivant RX. Applied logistic regression. Hoboken: Wiley, 2013: 1-500.

30 Vittinghoff E, Glidden DV, Shiboski SC, McCulloch CE. Regression methods in biostatistics: linear, logistic, survival, and repeated measures models. Boston: Springer, 2012.

31 Grambsch PM, Therneau TM. Proportional hazards tests and diagnostics based on weighted residuals. Biometrika 1994; 81: 515-26.

32 Koenker R. Quantile regression. Cambridge: Cambridge University Press, 2005.

33 R Core Team. R: a language and environment for statistical computing. Vienna: R Foundation for Statistical Computing, 2021.

34 Koenker R. quantreg: quantile regression. $\mathrm{R}$ package version 5.86. https://CRAN.R-project.org/package=quantreg (viewed July 2021).

35 Hagemann A. Cluster-robust bootstrap inference in quantile regression models. J Am Stat Assoc 2017; 112: 446-56.

36 European Medicines Agency Committee for Medicinal Products for Human Use. Guideline on adjustment for baseline covariates (draft). 2013. https://www.ema.europa.eu/docs/ en_GB/document_library/Scientific_guideline/2013/06/ WC500144946.pdf (viewed July 2021).

37 Jakobsen JC, Gluud C, Wetterslev J, Winkel P. When and how should multiple imputation be used for handling missing data in randomised clinical trials - a practical guide with flowcharts. BMC Med Res Methodol 2017; 17: 162.

38 Little RJ, D'Agostino R, Cohen ML, et al. The prevention and treatment of missing data in clinical trials. N Engl J Med 2012; 367: 1355-60.

39 Marshall A, Altman DG, Holder RL, Royston P. Combining estimates of interest in prognostic modelling studies after multiple imputation: current practice and guidelines. BMC Med Res Methodol 2009; 9: 57.

40 Geraci M. Estimation of regression quantiles in complex surveys with data missing at random: an application to birthweight determinants. Stat Methods Med Res 2016; 25: 1393-421.

41 Vidoni ML, Reininger BM, Lee M. A comparison of mean-based and quantile regression methods for analyzing self-report dietary intake data. J Probab Stat 2019; 2019: 9750538.

42 Angus DC, Derde L, Al-Beidh F, et al; Writing Committee for the REMAP-CAP Investigators. Effect of hydrocortisone on mortality and organ support in patients with severe COVID-19: the REMAP-CAP COVID-19 corticosteroid domain randomized clinical trial. JAMA 2020; 324: 1317-29.

43 Ananth CV, Kleinbaum DG. Regression models for ordinal responses: a review of methods and applications. Int J Epidemiol 1997; 26: 1323-33.

44 Armstrong BG, Sloan M. Ordinal regression models for epidemiologic data. Am J Epidemiol 1989; 129: 191-204.

45 Agresti A. Modelling ordered categorical data: recent advances and future challenges. Stat Med 1999; 18: 2191-207.

46 Brock GN, Barnes C, Ramirez JA, Myers J. How to handle mortality when investigating length of hospital stay and time 


\section{ORIGINAL ARTICLE}

to clinical stability. BMC Med Res Methodol 2011; 11: 144.

47 Wang R, Lagakos SW, Ware JH, et al. Statistics in medicine reporting of subgroup analyses in clinical trials. N Engl J Med 2007; 357: 2189-94.

48 Rockwood K, Song X, Macknight C, et al. A global clinical measure of fitness and frailty in elderly people. CMAJ 2005; 173: 489-95.

49 RECOVERY Collaborative Group; Horby P, Lim WS, Emberson JR, et al. Dexamethasone in hospitalized patients with Covid-19. N Engl J Med 2021; 384: 693-704.

50 Ustun TB, Kostanjesek N, Chatterji S, Rehm J; World Health Organization. Measuring health and disability: manual for WHO Disability Assessment Schedule (WHODAS 2.0). Geneva: WHO, 2010.

51 Knott RJ, Harris A, Higgins A, et al. Cost-effectiveness of erythropoietin in traumatic brain injury: a multinational trialbased economic analysis. J Neurotrauma 2019; 36: 2541-8.

52 Moher D, Hopewell S, Schulz KF, et al. CONSORT 2010 explanation and elaboration: updated guidelines for reporting parallel group randomised trials. BMJ 2010; 340: c869.

53 Ye C, Beyene J, Browne G, Thabane L. Estimating treatment effects in randomised controlled trials with non-compliance: a simulation study. BMJ Open 2014; 4: e005362.

54 Lehmann EL. Nonparametrics: statistical methods based on ranks. New York: Springer-Verlag, 2006.

55 ARISE Investigators; ANZICS Clinical Trials Group; Peake SL, Delaney A, Bailey $M$, et al. Goal-directed resuscitation for patients with early septic shock. N Engl J Med 2014; 371: 1496-506.

56 Jennison C, Turnbull BW. Group sequential methods with applications to clinical trials. Boca Raton: Chapman and Hall/ CRC, 2000: pp 1-390.

57 Cytel. East 6.5. Statistical software for the design, simulation and monitoring clinical trials. Cambridge: Cytel, 2018.

58 SAS Institute. SAS 9.4. Cary: SAS Institute, 2017.

59 Stata Corporation. Stata statistical software: release 16. 16.1 ed. College Station: StataCorp, 2019.

60 International Council for Harmonisation of Technical Requirements for Pharmaceuticals for Human Use. Integrated addendum to ICH E6(R1): guideline for good clinical practice E6(R2). 2016. https://database.ich.org/sites/default/files/E6_ R2_Addendum.pdf (viewed July 2021). 